\title{
Systematic review and meta-analysis of maternal secondhand smoke exposure and neonatal outcomes
}

\author{
Mark Parascandola', Amanda L. Klein², Julie Bromberg² \\ 'Tobacco Control Research Branch, Behavioural Research Program, Division of Cancer Control and Population Sciences, \\ National Cancer Institute, National Institutes of Health, Bethesda, MD, USA \\ ${ }^{2}$ BLH Technologies, Inc., Rockville, MD, USA
}

\begin{abstract}
Objectives: To determine the risk of adverse pregnancy and foetal outcomes among nonsmoking women exposed to secondhand smoke (SHS) while pregnant and compare the quality of studies in middle- and high-income countries (MIC and HIC).

Material and methods: To assess the effect of maternal SHS exposure on preterm birth, low birth weight, stillbirth, and orofacial clefts, the authors searched three databases (2004-2015) and applied strict inclusion/exclusion criteria. Crude odds ratios (ORs), adjusted ORs, associated 95\% confidence intervals, and raw data were used to estimate pooled ORs using random-effects models. Models were stratified by adjusted/unadjusted data, high study quality vs. low quality, and country income level.

Results: The authors identified 39 studies that assessed the effect of maternal SHS exposure on the four outcomes. Of these studies, the 20 conducted in MICs were of lower quality on average than those conducted in HICs. Evidence of a statistically significant effect of SHS exposure was found using adjusted pooled ORs among the high-quality studies for low birth weight (pOR: 1.282 [95\% CI: 1.103-1.490]), preterm birth (pOR: 1.290 [95\% CI: 1.127-1.476]), and stillbirth (pOR: 1.230 [95\% CI: 1.037-1.459]). Using the high-quality adjusted ORs, the effect of SHS exposure in nonsmoking pregnant women on cleft palate with or without cleft lip did not reach significance (pOR: 1.307 [95\% CI: 0.893-1.9]).

Conclusions: In all analyses, the odds of having negative outcomes were greater among studies conducted in MICs than in HICs. More research is needed in middle-income countries to inform policy and practice.
\end{abstract}

KEY WORDS: meta-analysis, second-hand smoke, reproductive health, pregnancy.

ADDRESS FOR CORRESPONDENCE: Mark Parascandola, PhD, MPH, Tobacco Control Research Branch, Behavioural Research Program, Division of Cancer Control and Population Sciences, National Cancer Institute, National Institutes of Health, 9609 Medical Centre Drive, Bethesda, MD, 20892, USA, e-mail: paramark@mail.nih.gov

\section{INTRODUCTION}

A large body of evidence demonstrates that cigarette smoking and secondhand smoke (SHS) exposure during pregnancy pose serious risks to the mother and her developing child, including sudden infant death syndrome, low birth weight (LBW), orofacial defects (cleft lip with/without cleft palate [CLP]), preterm birth (PTB), and stillbirth [1]. While attention has been paid to reducing cigarette smoking during pregnancy, especially in high-income countries (HICs), less progress has been made in reducing secondhand smoke exposure during pregnancy, especially in low- and middle-income countries (LMICs).

Substantial advances have been made over the past decade in implementing policies to reduce SHS exposure. Since 2007, 35 LMICs (25\% of all LMICs) have put in place comprehensive smoke-free laws. Overall, comprehensive smoke-free legislation is currently in place in 55 countries covering $20 \%$ of the world's population 
[2]. However, much of the world's population remains unprotected, and in countries where laws do exist, implementation and enforcement remain a challenge. Moreover, smoke-free policies generally target workplaces and public spaces, but not homes, so nonsmokers who live with a smoker remain affected. The World Health Organisation (WHO) estimates that 1.5 billion people are exposed to SHS at home, and 1.2 billion people are exposed to SHS in public places and workplaces [3].

In many LMICs, smoking rates among women remain low compared with men's rates. Thus, SHS exposure may be as important or more important to maternal and child health outcomes than active maternal smoking in these countries [4]. Of LMICs that have published rates of SHS exposure in women at home, rates are particularly high in China (63.9\%), Indonesia (75.4\%), Egypt (73.6\%), and Vietnam (69.2\%) [5]. In comparison, Caleyachetty et al., using nationally representative household Demographic and Health Surveys in 54 countries, estimated that overall prevalence of any form of tobacco use in pregnant women was $2.6 \%$ [6].

Six previous meta-analyses have been conducted looking at SHS exposure and maternal and child health outcomes. Four included LBW [7-10], two included PTB $[9,10]$, one included CLP $[11]$, and one included stillbirth [12]. An additional meta-analysis also analysed all orofacial clefts but did not conduct separate analyses for cleft lip [12]. A summary narrative of previous meta-analyses was published in 2013, which found, in general, a significant association between SHS exposure and LBW, PTB, and congenital malformations [13]. All of the mentioned meta-analyses found significantly increased odds of these health outcomes, with the exception of Salmasi et al. [10]. For LBW, PTB, and stillbirth, the most recent meta-analyses used 2009 data, and for orofacial clefts the most recent meta-analysis used 2013 data. However, additional studies have since been published, and previous analyses were limited primarily to studies conducted in HICs. For the purpose of this review, we evaluated studies focusing on the maternal and child health outcomes of low birth weight, preterm birth, orofacial cleft with or without cleft palate, and stillbirth, particularly seeking to include studies from LMICs.

\section{MATERIAL AND METHODS LITERATURE SEARCH STRATEGY - STUDY SELECTION}

Two reviewers (J.B. and A.H.) used separate, comprehensive strategies to search the PubMed, Embase, and Web of Science (Appendix 1) databases for publications relevant to smoking and risk of adverse pregnancy outcomes published between 2004 and December 2015. The reviewers independently screened the titles and abstracts of all citations identified in the search. The full-text article was retrieved if either reviewer considered the cita- tion potentially relevant. Disagreements or uncertainties were resolved by a third reviewer (M.P.).

\section{DATA ABSTRACTION}

Two reviewers extracted data on author, title, outcome reviewed, study design, case/control or event/ no-event data, crude odds ratios (ORs) and adjusted ORs (where presented), confounders, participant inclusion criteria, study sampling technique, and years of data included in the study. Differences in item coding were resolved by discussion.

\section{INCLUSION AND EXCLUSION CRITERIA}

Case-control, cohort, and cross-sectional studies were included if they reported on LBW, PTB, cleft lip/ palate, or stillbirth, as defined below, and compared mothers who were exposed to secondhand smoke with mothers who were not exposed, excluding mothers who were smokers. SHS exposure was determined either through self-report or via biochemical assay. Exposure could be domestic, occupational, or from other sources.

Studies were excluded if they: 1) were duplicates; 2) did not have at least English abstracts; or 3) did not account for maternal smoking or did not make clear whether active maternal smoking was a confounding factor. Experimental studies such as smoking cessation randomised control trials were also excluded.

\section{DEFINITION OF OUTCOMES}

Four main outcomes, which the 2014 Surgeon General's report [1] reported to be causally linked with smoking and/or SHS exposure, were chosen as the focus of this paper: - Low birth weight: A weight of less than $2500 \mathrm{~g}$ or 5 pounds 8 ounces at birth. Gestational age was not included in this definition because it was not always reported, particularly for lower quality studies.

- Preterm birth: A gestational age at birth of less than 37 weeks.

- Orofacial cleft (cleft lip with or without cleft palate): A clinical diagnosis of orofacial cleft lip with or without cleft palate, excluding any participants with a chromosomal abnormality or syndrome.

- Stillbirth: Birth of an infant that died in the womb after 20 weeks of pregnancy as determined by ultrasound, or ultrasound plus last menstrual period, and/or a minimum weight for gestational age (i.e. $400 \mathrm{~g}$ at 24 weeks).

\section{DEFINITION OF EXPOSURES}

Studies that separated the mother's SHS exposure from the mother's active smoking were eligible for inclusion. Studies were included if they described the SHS exposure, which could be defined by biochemical validation or measuring air quality, or by mothers' self-report of: whether they were exposed (yes or no), the amount of smoke exposure in hours or minutes per day, the number of cigarettes per day that were smoked around them, or the source of 
exposure (i.e. paternal, spouse, other). For biochemical validation, serum cotinine at acceptable levels was used to determine nonsmokers not exposed to SHS (under $0.01 \mathrm{ng} / \mathrm{ml}$ ). For exposures where odds ratios were calculated for different amounts of exposure, the odds ratios were used for the maximum exposure time. For exposures that measured time in pregnancy (early vs. late), early pregnancy odds ratios were used. For both exposures, the ORs were chosen to increase the chances of seeing an effect of SHS on maternal and child outcomes.

\section{QUALITY ASSESSMENT}

Study quality was assessed using an adapted NewcastleOttawa Scale - a nine-point scale assessing exposed (cases) and unexposed (controls) selection, confounding variables, measurement of secondhand smoke, and nonresponse rates. Two reviewers coded all eligible studies for quality.

\section{ANALYSIS}

Tabulated data, crude estimates, and/or adjusted estimates were extracted from the included studies. A random effects model was chosen to account for heterogeneity of study populations and designs. Multiple separate analyses were conducted for each outcome: unadjusted full sample using either raw data or crude ORs, unadjusted high-quality sample using only studies that had a quality rating over 6 , adjusted full sample using aORs to account for confounders, and adjusted high-quality studies. Additionally, studies were stratified by country income group (HIC vs. MIC) for each outcome. Analy- ses included adjusted odds ratios to account for potential confounders, despite individual studies adjusting for different factors. Pooled odds ratios (pORs) and 95\% confidence intervals were calculated for each analysis.

Heterogeneity between studies was assessed using the $I^{2}$ statistic, which represents the percentage of total variation that is attributable to between-studies heterogeneity. Statistical significance of heterogeneity was analysed with the Q statistic. If $I^{2}$ exceeded $75 \%$, separate sensitivity analyses were conducted to determine the cause of this high heterogeneity. Data were analysed using the comprehensive meta-analysis software. Probability values below 0.05 were considered statistically significant.

\section{RESULTS}

\section{LITERATURE SEARCH}

Figure 1 shows the article-screening process. Of the identified publications, 5470 unique publications remained after duplicates were removed. Title and abstract screening resulted in exclusion of 4947 records, primarily due to irrelevance. Studies were then sorted by topic-LBW, PTB, CLP, and stillbirth, resulting in 198 remaining records. Finally, based on full text analysis, studies not meeting the exclusion criteria were removed, resulting in 39 studies included.

\section{STUDY CHARACTERISTICS AND QUALITY ASSESSMENT}

Table 1 summarises the included studies [14-52]. An additional 13 studies were identified for LBW [14-25],

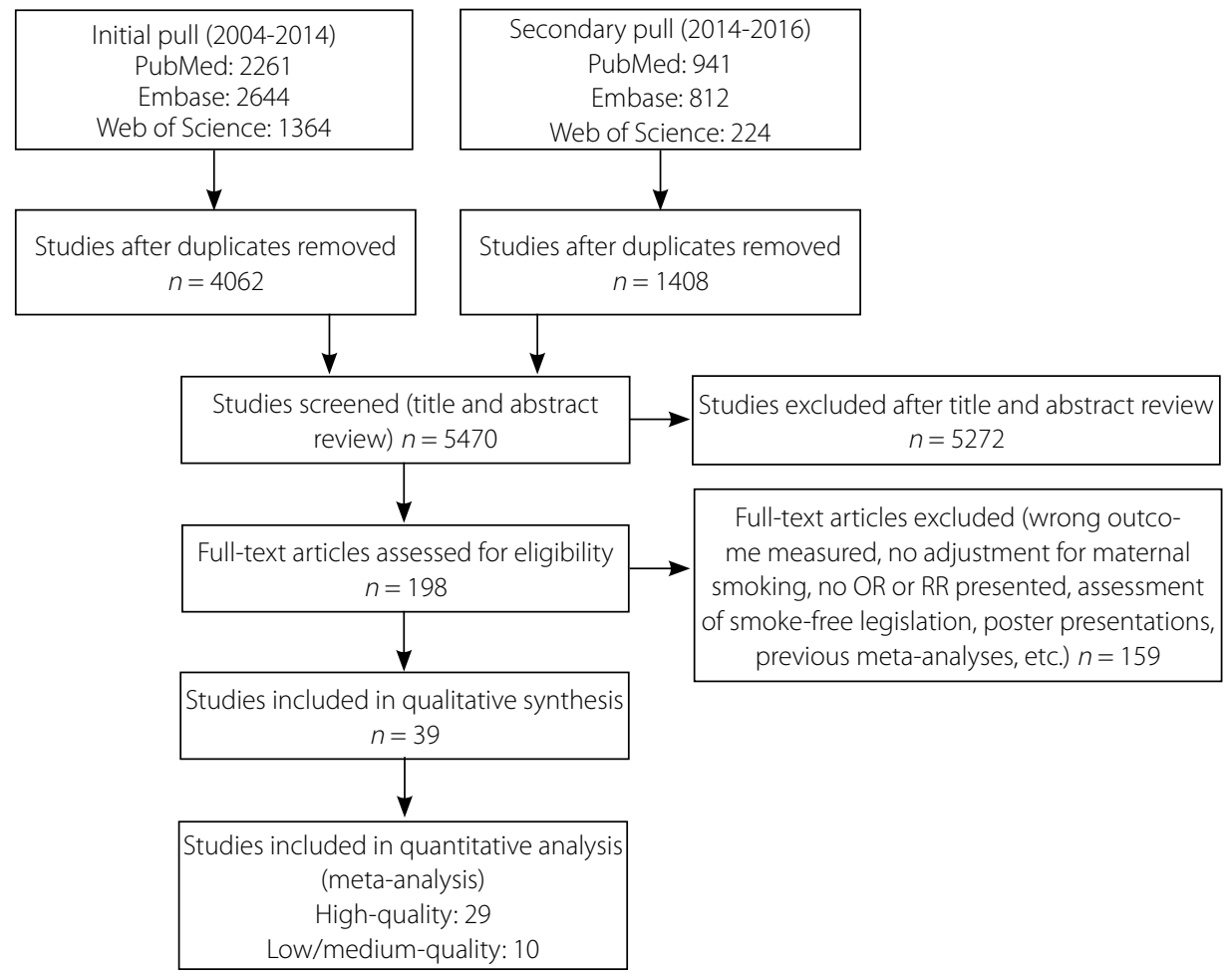

FIG. 1. Prisma diagram of study selection 


\begin{tabular}{|c|c|c|c|c|c|c|c|c|c|c|c|c|}
\hline$\delta$ & $\wedge$ & $\infty$ & 0 & 0 & $\infty$ & in & $\wedge$ & 0 & $\wedge$ & 0 & $\sigma$ & in \\
\hline 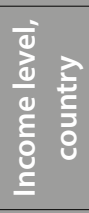 & $\begin{array}{l}\frac{c}{0} \\
\frac{0}{0} \\
\frac{0}{0} \\
\grave{5}\end{array}$ & $\begin{array}{l}\sqrt[J]{N} \\
\mathbb{N} \\
\sum \\
\Sigma\end{array}$ & 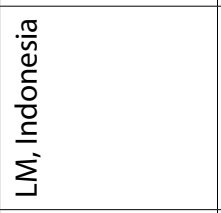 & 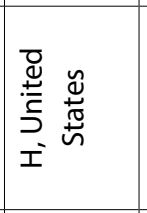 & 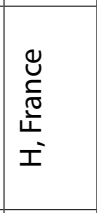 & $\begin{array}{l}\frac{\pi}{0} \\
\frac{0}{0} \\
\tilde{N} \\
I \\
I\end{array}$ & $\begin{array}{l}\frac{\lambda}{\overline{\bar{T}}} \\
\frac{1}{I} \\
\end{array}$ & 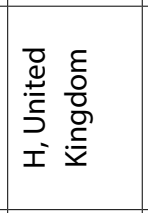 & 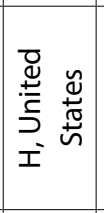 & 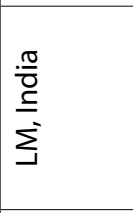 & 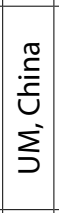 & 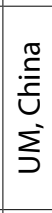 \\
\hline 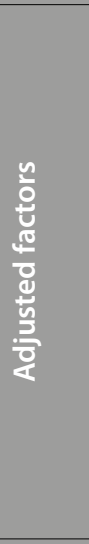 & 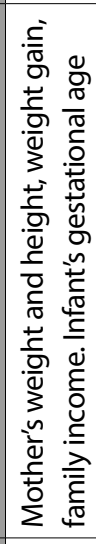 & 1 & 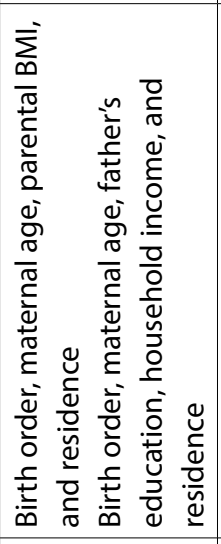 & 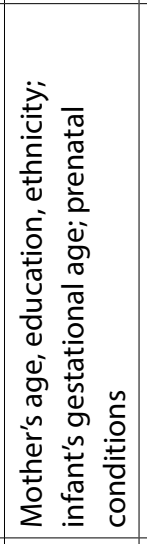 & 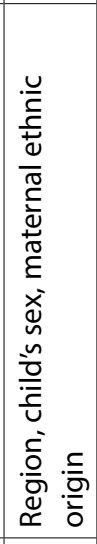 & 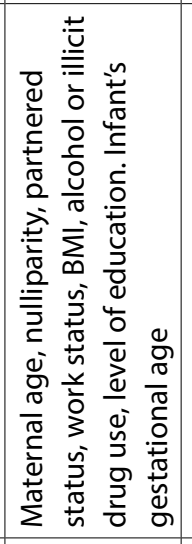 & 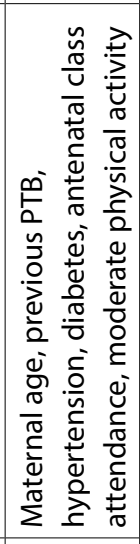 & 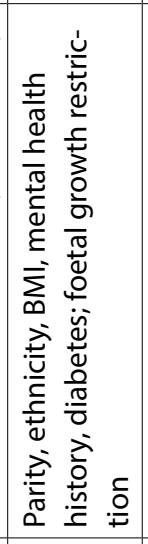 & 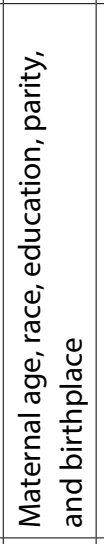 & 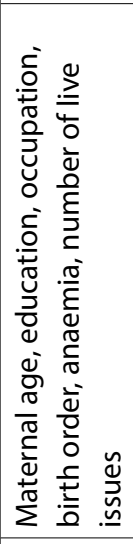 & 1 & 1 \\
\hline 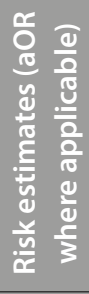 & 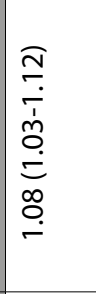 & 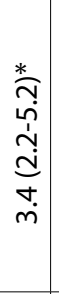 & 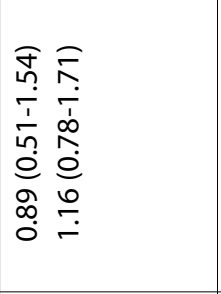 & & 1 & 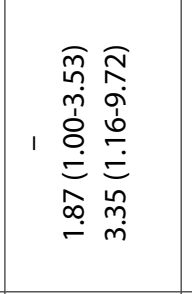 & 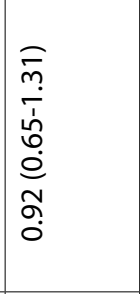 & 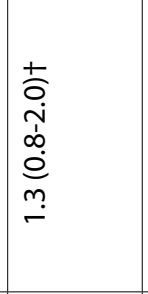 & 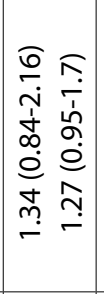 & 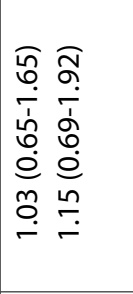 & 1 & 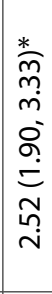 \\
\hline 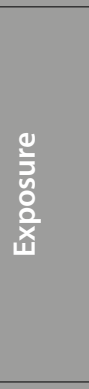 & 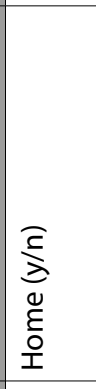 & 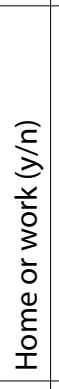 & 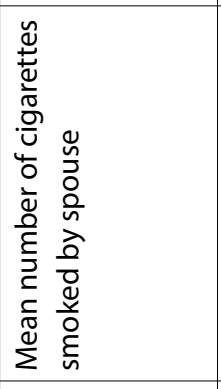 & 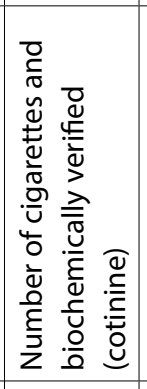 & 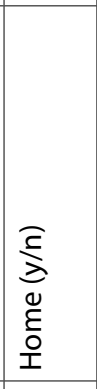 & 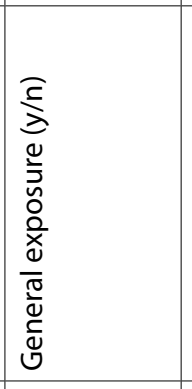 & 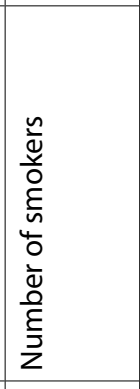 & 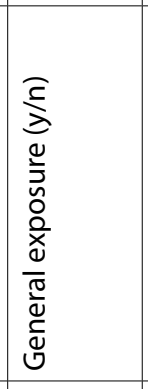 & 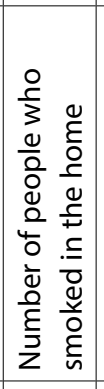 & 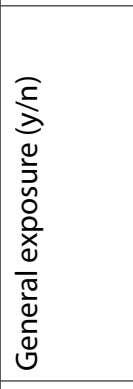 & 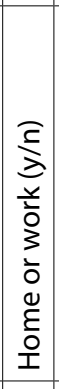 & 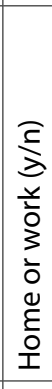 \\
\hline 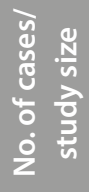 & ষ্ণ & $\stackrel{\mathscr{f}}{f}$ & $\frac{\sigma}{m} \frac{\alpha}{m}$ & 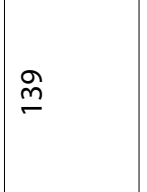 & $\stackrel{\infty}{\sim}$ & 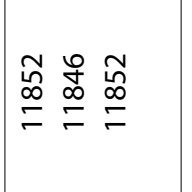 & 苑 & 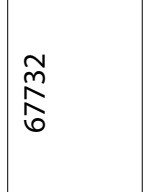 & 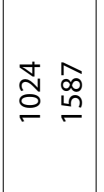 & $\begin{array}{ll}0 \\
i n \\
i n\end{array}$ & ষ্ণ & $\underset{\infty}{\mathcal{Y}}$ \\
\hline 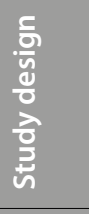 & 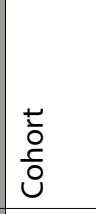 & 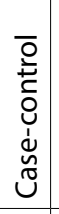 & $\begin{array}{l}\frac{\pi}{0} \\
\frac{\pi}{0} \\
\end{array}$ & 岕 & 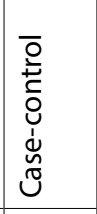 & $\begin{array}{l}\frac{\pi}{0} \\
\frac{1}{0} \\
0\end{array}$ & 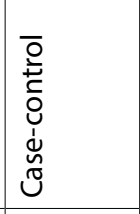 & $\begin{array}{l}\frac{\pi}{0} \\
\frac{1}{0} \\
0\end{array}$ & 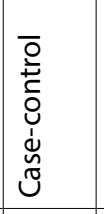 & $\begin{array}{l}\pi \\
0 \\
\frac{\pi}{0} \\
0\end{array}$ & 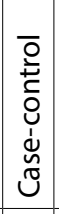 & 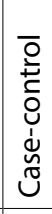 \\
\hline 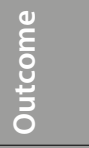 & 㐫 & 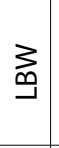 & 葛吕 & $\frac{\rho}{a}$ & ปे & 葛占喜 & $\stackrel{\infty}{\underline{L}}$ & 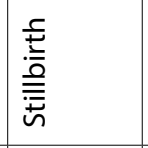 & 䓪吕 & 葛吕 & 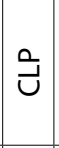 & 己े \\
\hline 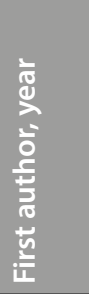 & 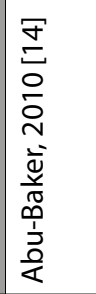 & 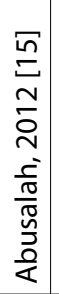 & 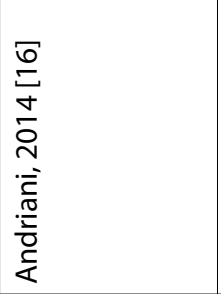 & 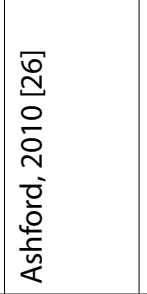 & 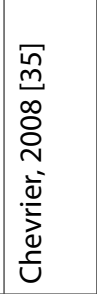 & 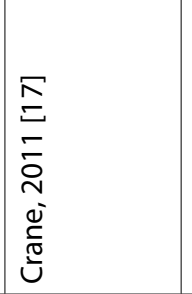 & 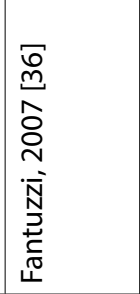 & 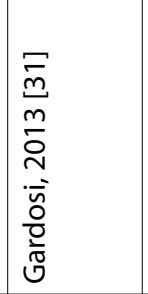 & 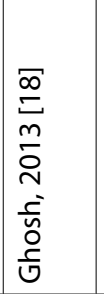 & 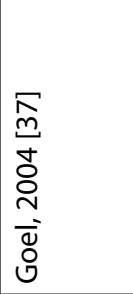 & 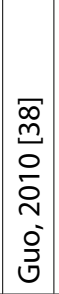 & 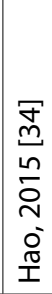 \\
\hline
\end{tabular}




\begin{tabular}{|c|c|c|c|c|c|c|c|c|c|c|c|}
\hline 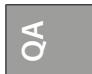 & 0 & r & $\infty$ & n & in & 0 & 0 & $\wedge$ & $\wedge$ & $r$ & 0 \\
\hline 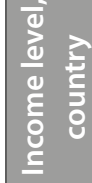 & 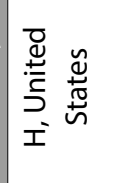 & 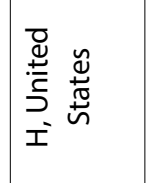 & 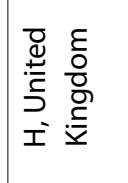 & 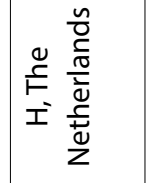 & 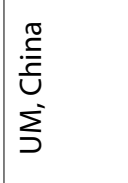 & 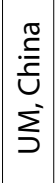 & 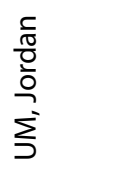 & 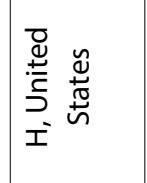 & 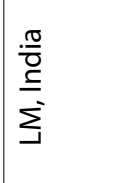 & 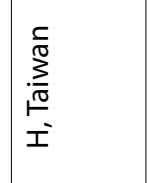 & 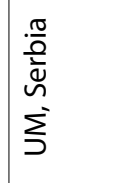 \\
\hline 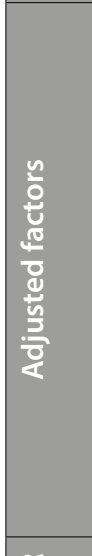 & 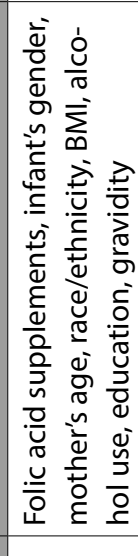 & 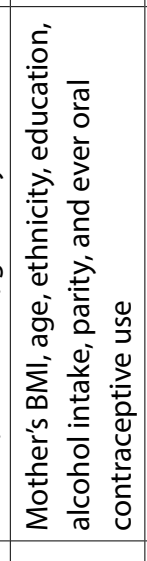 & 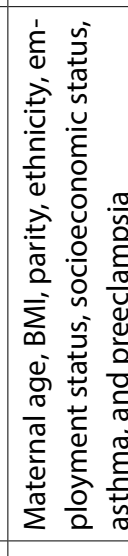 & 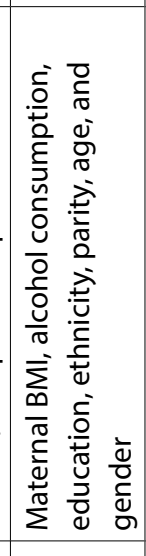 & 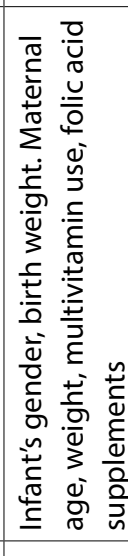 & 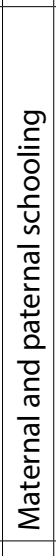 & 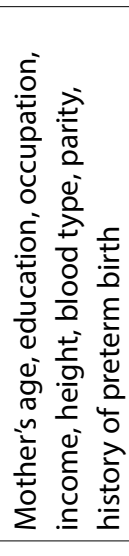 & . & 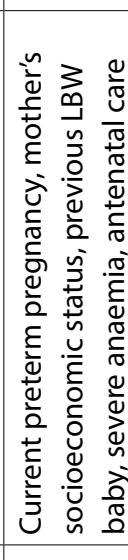 & 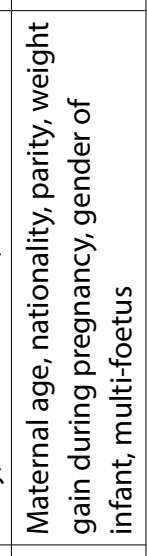 & 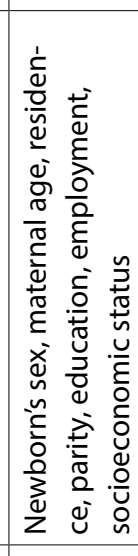 \\
\hline 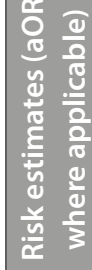 & $\begin{array}{l}\widehat{m} \\
\bar{\infty} \\
\stackrel{\infty}{0} \\
\stackrel{0}{\circ} \\
\stackrel{0}{c}\end{array}$ & 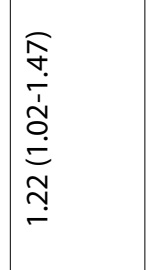 & 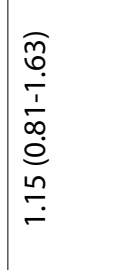 & 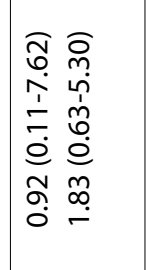 & 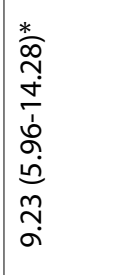 & 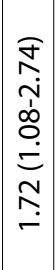 & 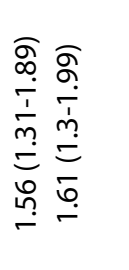 & I & 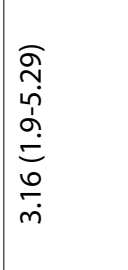 & 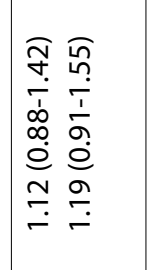 & 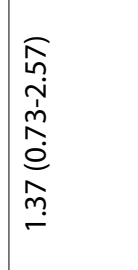 \\
\hline $\begin{array}{l}\text { 言 } \\
\text { वें }\end{array}$ & 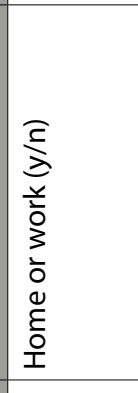 & 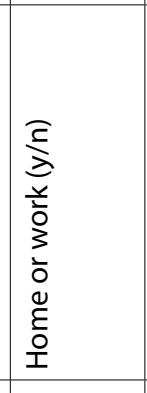 & 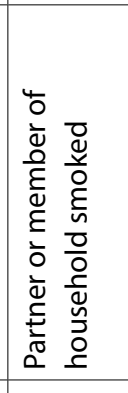 & 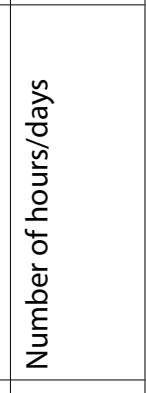 & 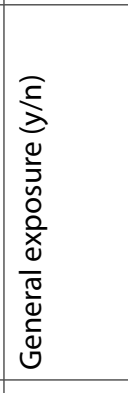 & 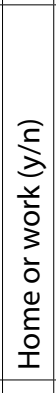 & 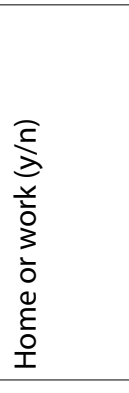 & 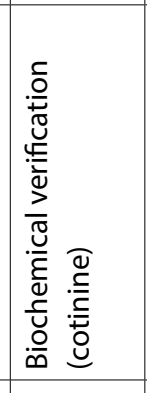 & 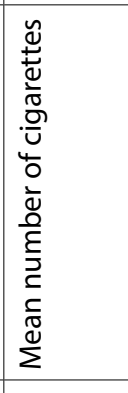 & 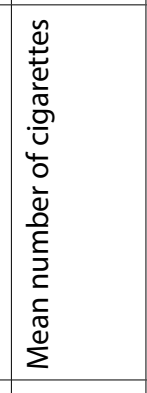 & 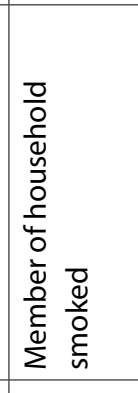 \\
\hline 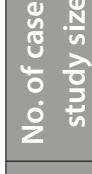 & $\underset{\widetilde{F}}{\widetilde{\gamma}}$ & 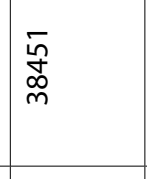 & $\begin{array}{l}\alpha \\
\hat{O} \\
\underline{m}\end{array}$ & $\begin{array}{l}\infty \\
\vdots \\
\vdots \\
2\end{array}$ & $\stackrel{\circ}{N}$ & \&্ণ & 鉣卓 & 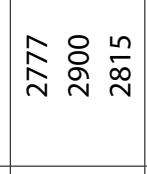 & 品 & 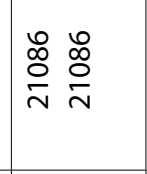 & fo \\
\hline 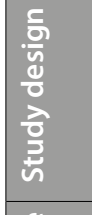 & 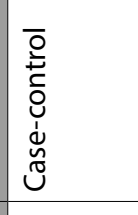 & $\begin{array}{l}\frac{t}{0} \\
\frac{0}{0} \\
0\end{array}$ & $\begin{array}{l}\frac{t}{0} \\
\frac{0}{3} \\
\end{array}$ & $\begin{array}{l}\frac{t}{0} \\
\frac{5}{0} \\
0\end{array}$ & 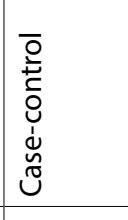 & 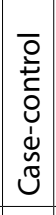 & 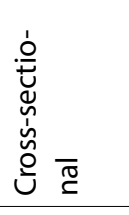 & $\begin{array}{l}\frac{5}{0} \\
\text { o } \\
\text { b }\end{array}$ & 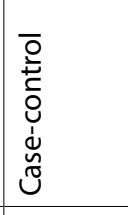 & 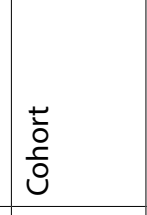 & $\begin{array}{l}\text { to } \\
\text { o. } \\
0\end{array}$ \\
\hline छू & ปे & 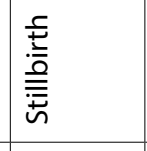 & 点 & 艿魚 & ปे & ปे & 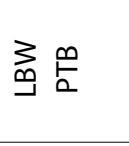 & 各畗喜 & 举 & 艿魚 & 孞 \\
\hline . & 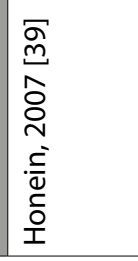 & 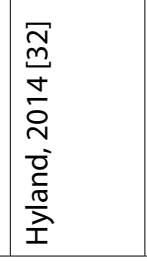 & 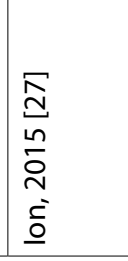 & 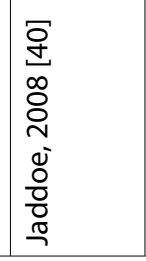 & 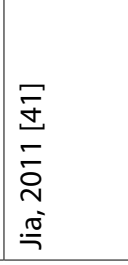 & 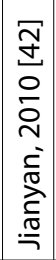 & 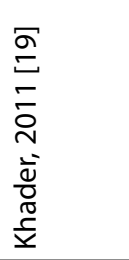 & 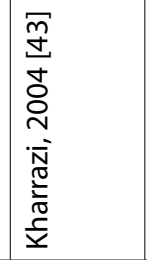 & 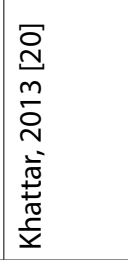 & 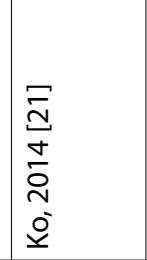 & 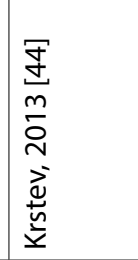 \\
\hline
\end{tabular}




\begin{tabular}{|c|c|c|c|c|c|c|c|c|c|c|c|}
\hline$\delta$ & 0 & 0 & $a$ & $m$ & $m$ & 0 & in & 0 & N & o & 。 \\
\hline & 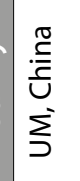 & 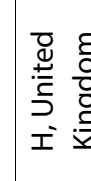 & 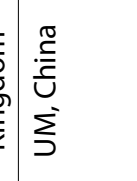 & 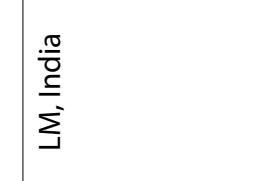 & 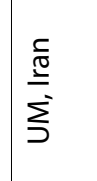 & 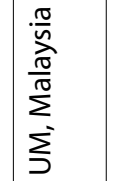 & 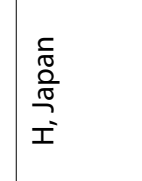 & 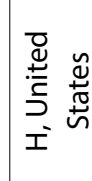 & 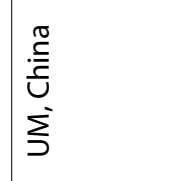 & 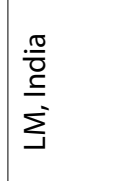 & 离 \\
\hline & 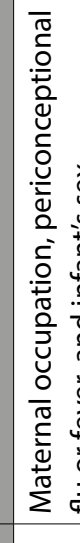 & 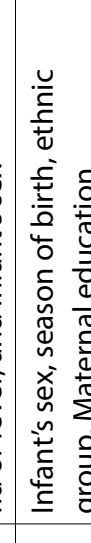 & 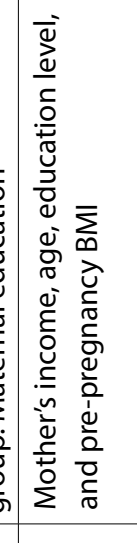 & 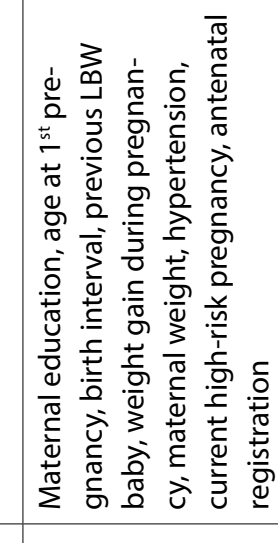 & , & 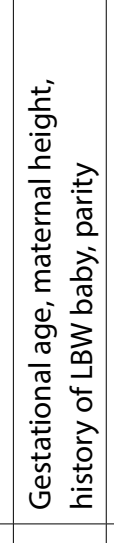 & 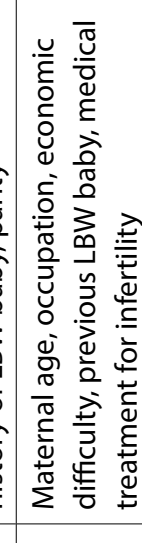 & 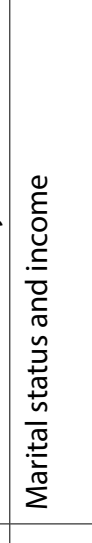 & 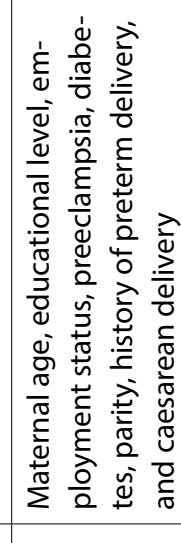 & & 1 \\
\hline & 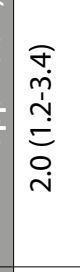 & 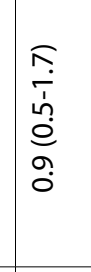 & 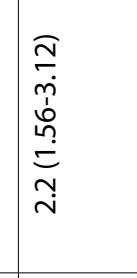 & 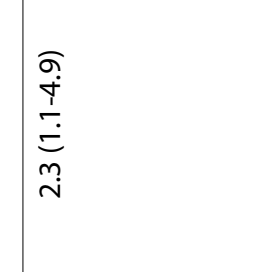 & 1 & 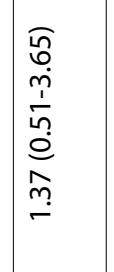 & 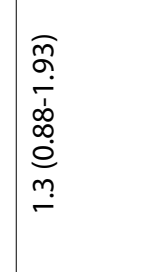 & 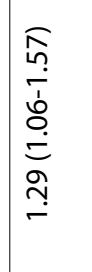 & 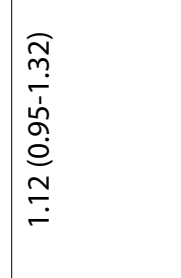 & ' & 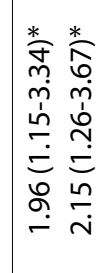 \\
\hline 啇 & 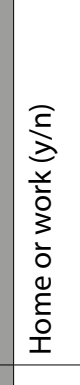 & 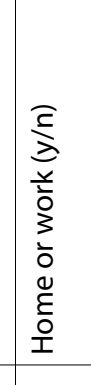 & 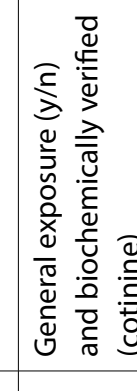 & 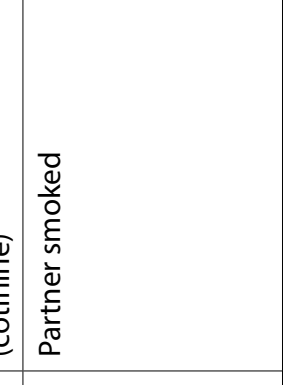 & 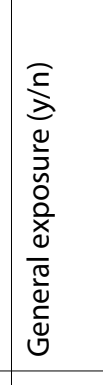 & 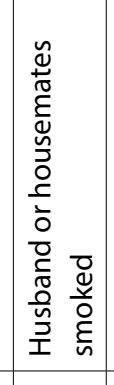 & 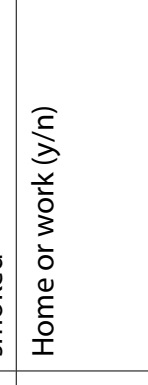 & 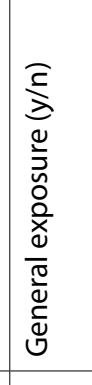 & 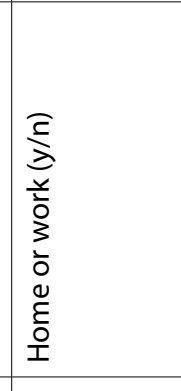 & 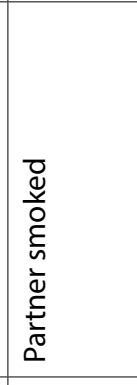 & 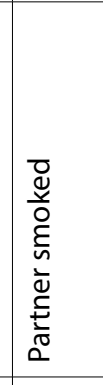 \\
\hline & iे & $\stackrel{\circ}{N}$ & $\approx$ & $\stackrel{\substack{m \\
=}}{\stackrel{2}{n}}$ & $\stackrel{\text { ă }}{\sim}$ & $\stackrel{乛}{\not}$ & 용 & : & 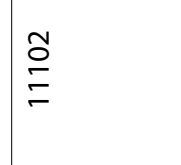 & 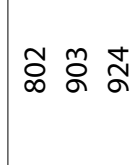 & 学学 \\
\hline & 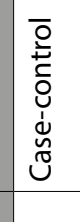 & 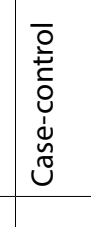 & 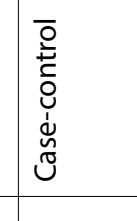 & $\begin{array}{l}\frac{\mathrm{t}}{\frac{5}{b}} \\
\frac{1}{2}\end{array}$ & 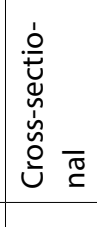 & $\frac{5}{\frac{5}{g}}$ & 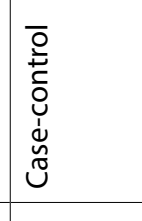 & $\begin{array}{l}\frac{5}{0} \\
\text { oj }\end{array}$ & $\begin{array}{l}\frac{\overline{0}}{0} \\
\frac{0}{3}\end{array}$ & $\begin{array}{l}\frac{5}{0} \\
\frac{0}{9} \\
\end{array}$ & $\begin{array}{l}\frac{\mathrm{t}}{\frac{0}{9}} \\
\end{array}$ \\
\hline & ปे & Uे & $\underline{m}$ & 童 & $\underline{\varrho}$ & 夢 & 莤 & 㱏 & 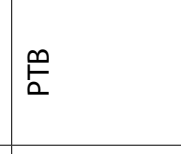 & 产吕旁害 & 总鱼 \\
\hline & 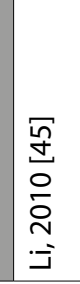 & 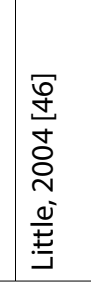 & 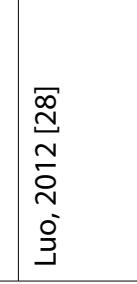 & 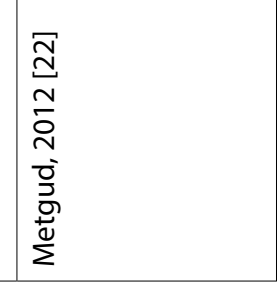 & 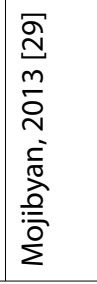 & 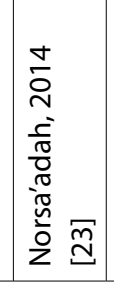 & 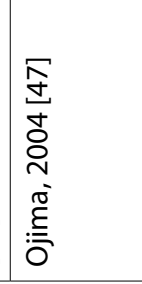 & 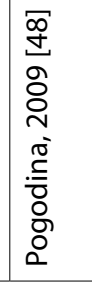 & 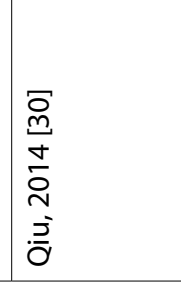 & 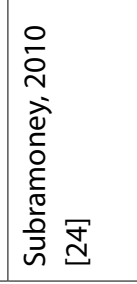 & 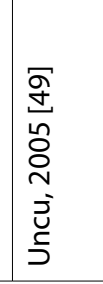 \\
\hline
\end{tabular}




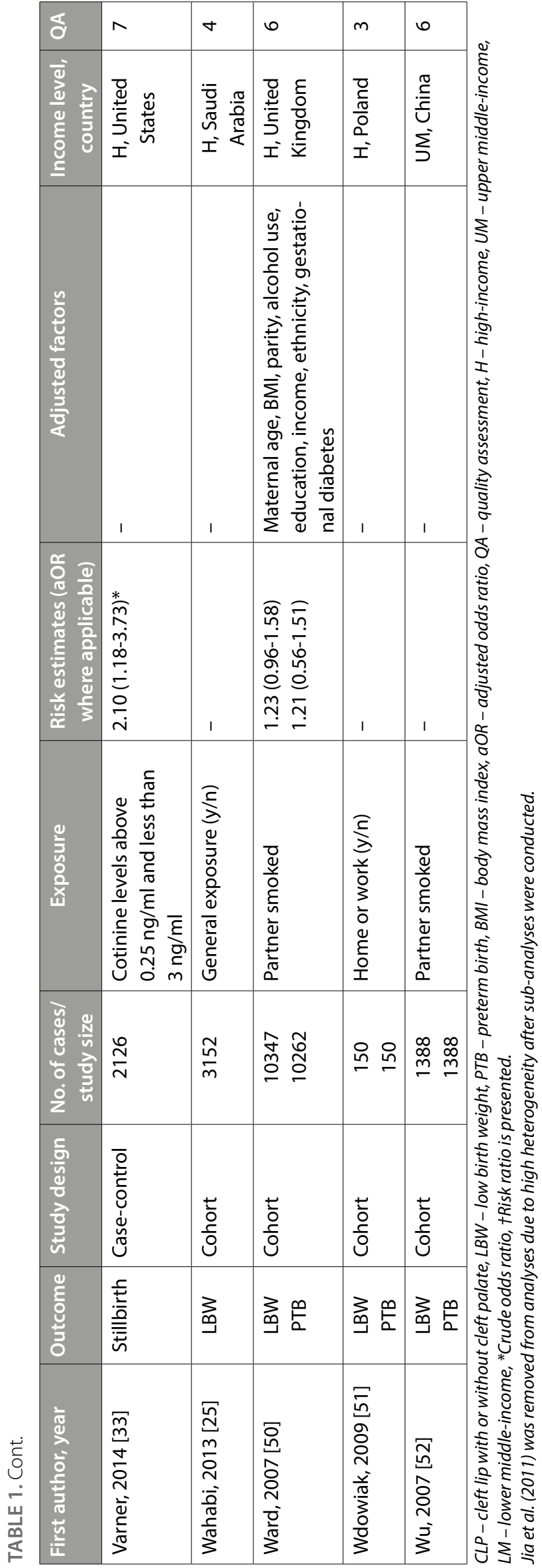

11 for PTB [16-19, 21, 24, 26-30], five for stillbirth $[17,24,31-33]$, and one for cleft lip [34], beyond those included in previous reviews. Together, the 39 studies represented 223,073 women/infants: 78,948 were included in LBW analyses; 89,831 in PTB analyses; 123,900 in stillbirth analyses; and 7422 in cleft lip analyses (some women were included in multiple analyses). SHS exposure was collected via cotinine assay in two studies [33, 43 ] and via self-reports and cotinine assay in two studies $[26,28]$. All other SHS measures were self-reported. Two studies measured SHS exposure in the home, 12 studies measured exposure at home or at work, 11 studies measured exposure to smoking by a partner or spouse, and 14 studies did not identify the source of the SHS exposure.

Of the 39 studies, 19 (49\%) were conducted in HICs, 14 were conducted in upper middle-income countries, and six were conducted in lower middle-income countries. For this review, no studies were identified that occurred in low-income countries. Study quality ratings ranged from 3.0 to 9.0 , with a mean quality score of 6.0 . The mean quality score for studies from HICs was 6.2; for those from lower MICs, it was 6.0; and for studies from upper MICs, it was 5.7. Twenty-nine studies (74\%) were considered to be of high quality, having scores higher than the chosen threshold of 6.0. The quality of other studies was lower due to a number of factors including inadequately ascertaining SHS exposure or adjusting for confounders. The results of the meta-analyses are presented in Table 2.

\section{OUTCOME MEASURES}

\section{Low birth weight}

The effect of SHS exposure on the risk of low birth weight was assessed using 15 cohort/cross-sectional studies and four case-control studies in the unadjusted analyses. Fourteen studies presented aORs, three of which did not include unadjusted ORs and thus were not included in both analyses (Fig. 2). Studies were conducted in 16 countries, including three lower MICs (Gaza, India, and Indonesia) and five upper MICs (China, Jordan, Malaysia, Serbia, and Turkey); studies from these 16 countries had a mean quality assessment score of 5.8 . All analyses were significant: Overall exposure to SHS was associated with a $28 \%$ increase in the risk of delivering a low birth weight infant $(n=12$; 95\% CI: 1.103 $1.490)$. Results using the adjusted high-quality sample $(\mathrm{QA}>6)$ in MICs resulted in pORs higher than in the analyses of all countries but still highly significant $(n=7$; pOR: 1.351 [95\% CI: 1.029-1.773]).

\section{Preterm birth}

The effect of maternal SHS exposure on the risk of preterm birth was assessed using 14 cohort/cross-sectional studies and three case-control studies in the unadjusted analyses (Fig. 3). Thirteen studies were included in the adjusted analyses, including two studies that only 
TABLE 2. Meta-analysis results

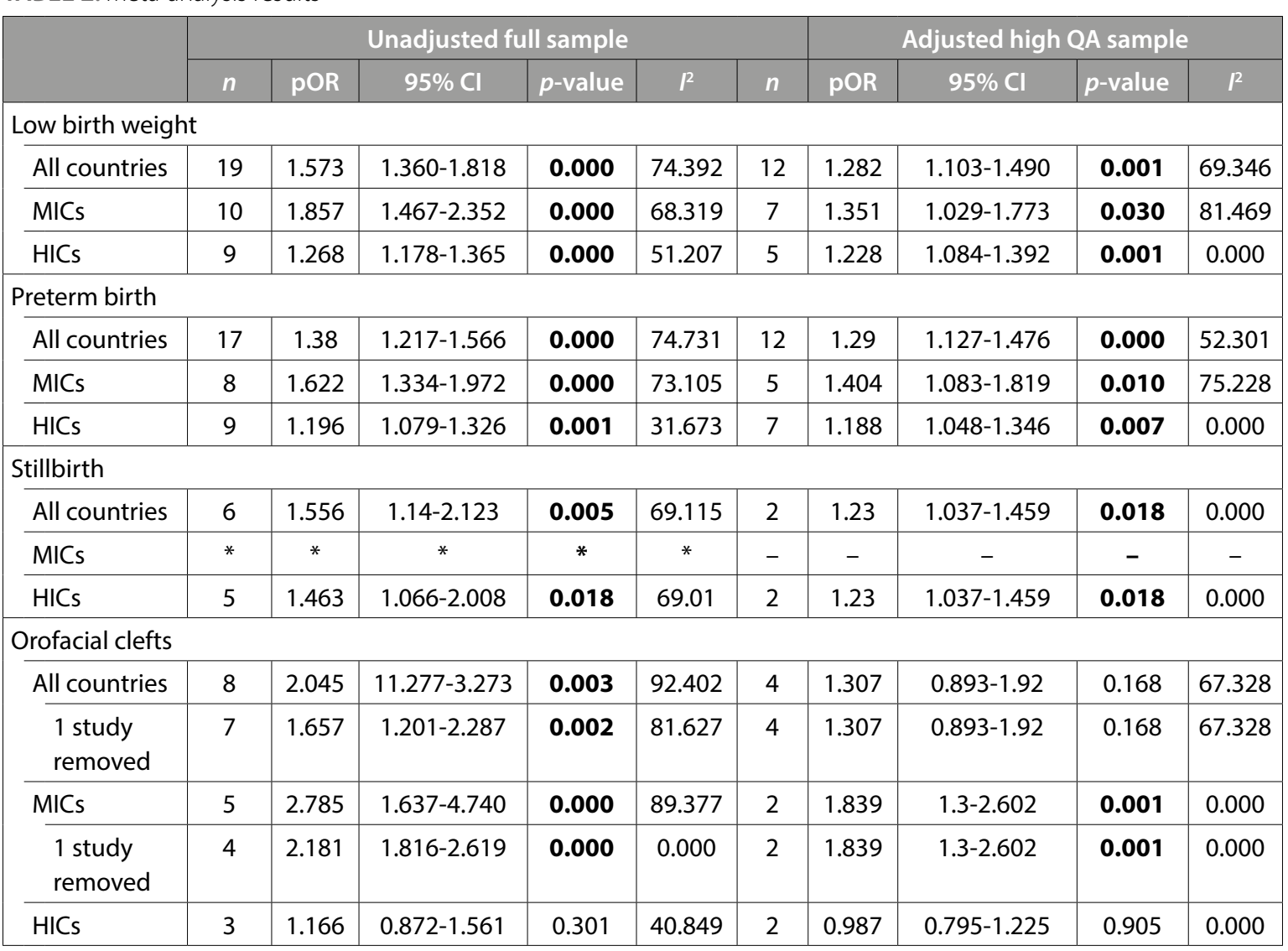

pOR - pooled odds ratio, Cl - confidence interval, MIC - middle-income country, HIC - high-income country. *one study fit criteria, no metaanalysis conducted. Bold $p$-values are significant at the level $p=0.05$.

presented aORs. Studies were conducted in 19 countries, including two lower MICs (India and Indonesia) and four upper MICs (China, Iran, Jordan, and Turkey); studies in these 19 countries had a mean QA score of 6.1. All analyses were significant. Overall exposure to SHS was associated with a $29 \%$ increase in the risk of delivering an infant preterm ( $n=12$; 95\% CI: 1.127-1.476). Results using a high quality-adjusted (QA $>6$ ) sample in MICs resulted in pORs higher than in the analyses of all countries and still highly significant $(n=7$; pOR: 1.404 [95\% CI: 1.083-1.819]).

\section{Stillbirth}

The effect of SHS exposure on the risk of stillbirth was assessed in five cohort/cross-sectional studies and one case-control study (Fig. 4). Three of the six studies were also included in adjusted analyses. Studies were conducted in four countries, including one lower middle-income country (India), and had a mean QA score of 6.3. Due to only one study in an MIC, analyses could not be stratified by country income group. All results were significant except for the sub-analysis using only adjusted data. Using adjusted high-quality studies, SHS exposure was associated with a $23 \%$ increase in the risk of having a stillbirth $(n=2 ; 95 \%$ CI: 1.037-1.459).

\section{Orofacial clefts}

Eight studies were included in the unadjusted CLP analyses, of which four studies were included in the adjusted analyses, all of which were case-controls. Three studies were conducted in HICs, and five were conducted in China (an upper middle-income country). These eight studies had a mean QA score of 5.75. $I^{2}$ values were extremely high for this analysis. By removing individual studies from the analysis, it was determined that the Jia et al. [41] study was driving the heterogeneity; for this reason, it was removed from analyses of the full sample, and due to low QA it was not included in the statistics presented below or in Figure 5. Analyses of high-quality studies show that SHS exposure was associated with a $31 \%$ increase in the odds of CLP $(n=4,95 \%$ CI: 0.893 1.92) (Fig. 5). Studies from MICs alone show that exposure to SHS is associated with an $84 \%$ increased risk ( $n=2$; 95\% CI: 1.30-2.602).

\section{DISCUSSION}

Consistent with previous meta-analyses, a significant increase in odds of LBW, PTB, and stillbirth associated with SHS exposure was found in both the full sample and in studies from HICs and MICs. Unlike previous meta-analyses, the current work included studies 


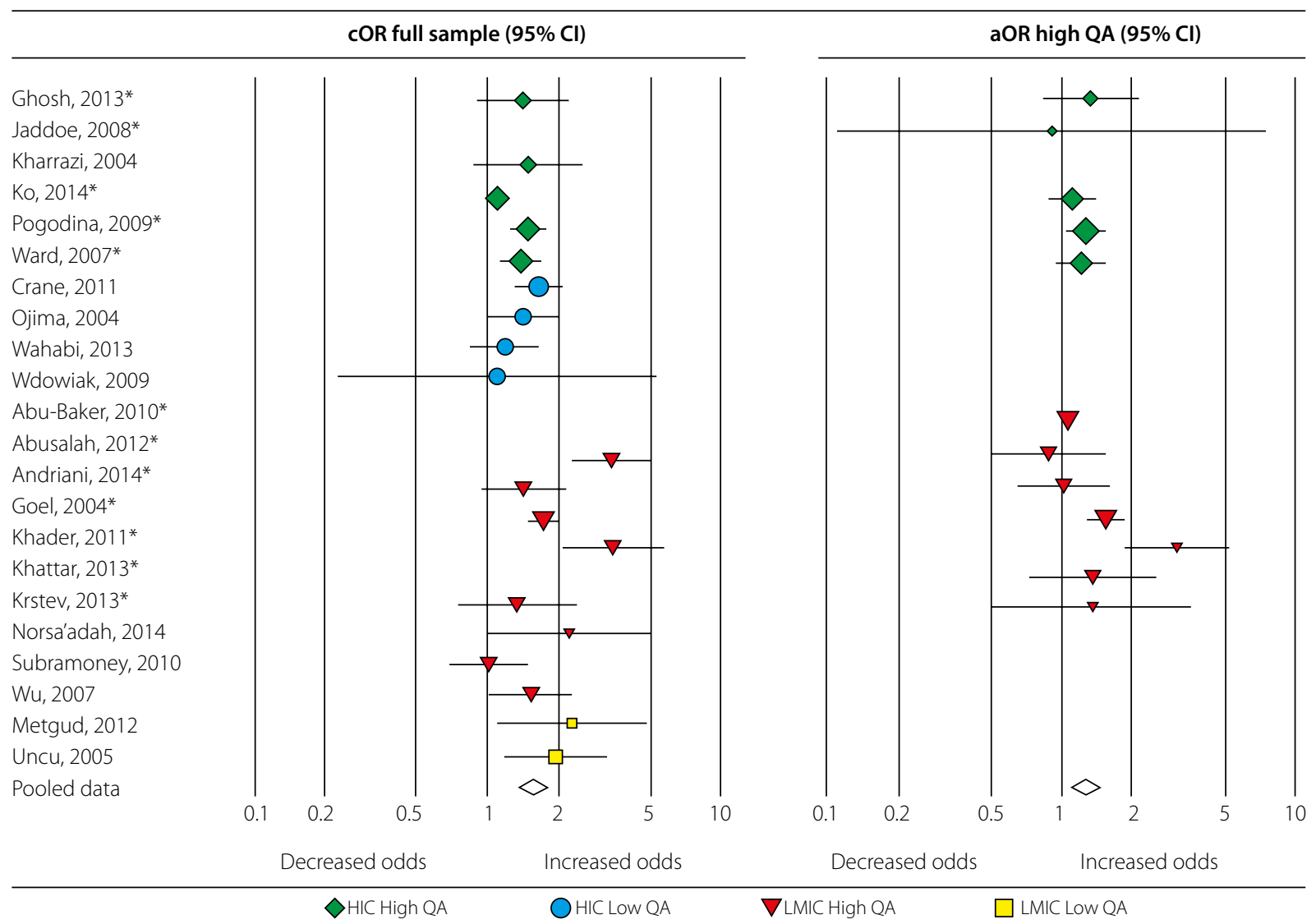

cOR - crude odds ratio, aOR - adjusted odds ratio, *adjusted odds ratios included in high-quality analysis, $\mathrm{Cl}$ - confidence interval,

QA - quality assessment, HIC - high-income country, LMIC - low- or middle-income country

FIG. 2. Low birth weight forest plot

from MICs. For LBW, previous meta-analyses found pORs between 1.17 and 1.60. In the current meta-analysis, findings for both the unadjusted full sample (pOR $=1.573$ ) and the adjusted high-quality sample (pOR = 1.282) fall within this range. The findings for stillbirth among the adjusted high-quality studies in this analysis ( $\mathrm{pOR}=1.23$ ) were comparable with previous meta-analyses; however, this analysis was limited to HICs because of the lack of studies assessing stillbirth from LMICs. The odds of PTB among the high-quality studies were higher in our analysis ( $\mathrm{pOR}=1.29$ across all studies; $\mathrm{pOR}=1.4$ in MICs) than in previous meta-analyses ( $\mathrm{pOR}=1.16$ 1.18). The current meta-analysis also found that odds for CLP using the unadjusted data $(\mathrm{pOR}=2.045)$ were consistent with previous reports ( $\mathrm{pOR}=2.05)$ [11] but were much lower and not statistically significant when using the adjusted high-quality sample ( $\mathrm{pOR}=1.307$ ). The current meta-analysis found that studies in the MIC group resulted in higher pORs than those for HICs (for example, 1.4 for preterm birth in MICs vs. 1.19 in HICs). This trend holds true for all sub-analyses conducted. These differences may be due to greater exposure levels and weaker protections from SHS in MICs, differences in smoked products and patterns of use, or possible interactions with other reproductive health risks. However, given that there are still relatively few high-quality studies from LMICs, future studies should further explore these differences.

Substantial uncertainty remains around the extent of the burden on maternal and child health from SHS exposure, particularly in low- and middle-income countries. One survey of nine developing countries reported that SHS exposure during pregnancy ranged widely, from $17.1 \%$ in the Democratic Republic of Congo to $91.6 \%$ in Pakistan, but comprehensive data on SHS exposure during pregnancy is lacking from most LMICs $[53,54]$. Although data is limited, estimates suggest that more than one-third of nonsmoking women (35\%) of reproductive age could be exposed to SHS in LMICs [55]. Thus, among women and children in many LMICs, the disease burden from SHS exposure may equal to or exceed that from direct smoking. Additionally, within LMICs, exposure to SHS may be even greater among groups with lower socioeconomic status, because low educational attainment and low wealth 


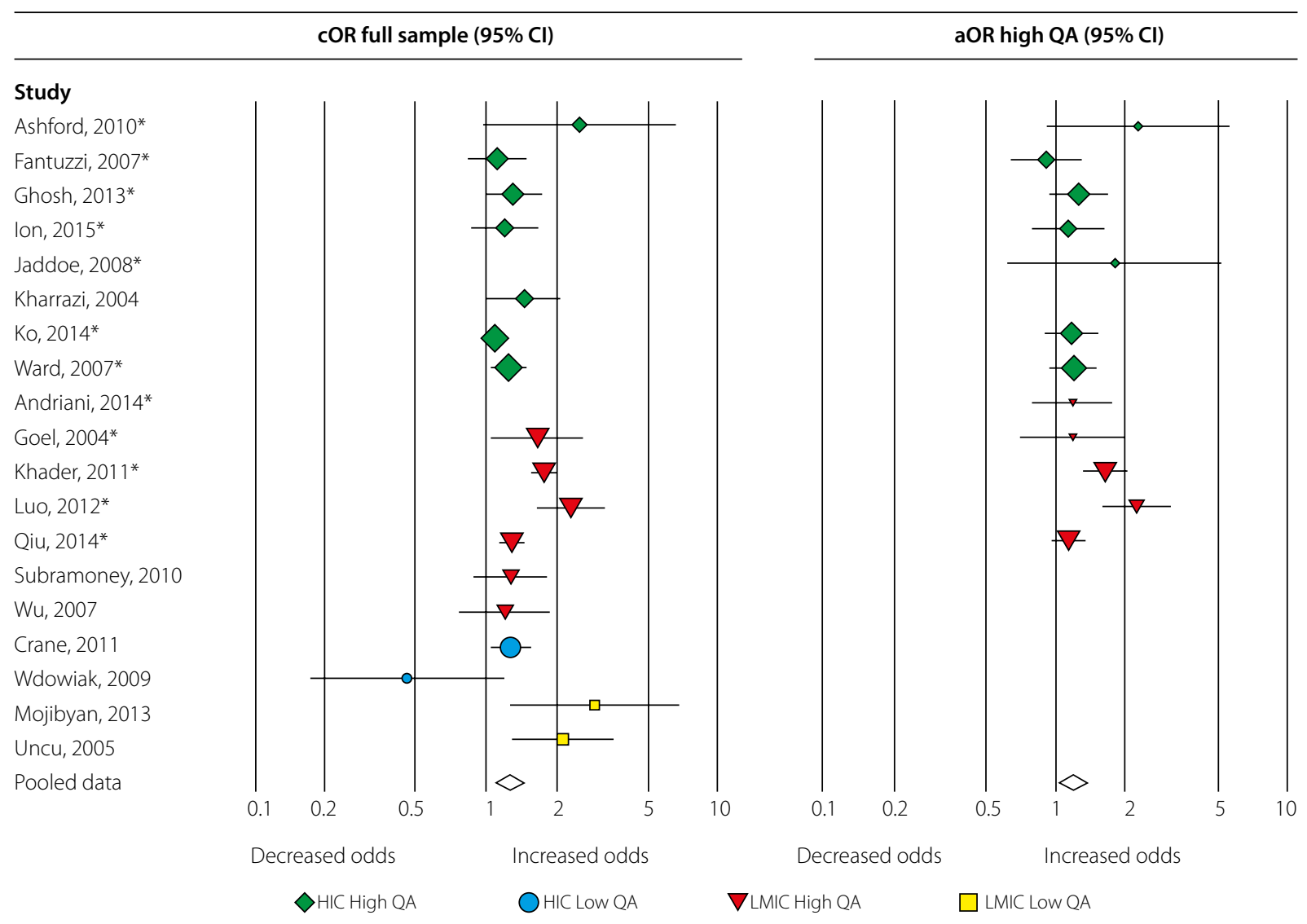

COR - crude odds ratio, aOR - adjusted odds ratio, *adjusted odds ratios included in high-quality analysis, Cl - confidence interval, QA - quality assessment, HIC - high-income country, LMIC - low- or middle-income country

FIG. 3. Preterm birth forest plot

cOR full sample $(95 \% \mathrm{Cl})$

\section{Study}

Gardosi, 2013*

Hyland, 2014*

Kharrazi, 2004

Varner, 2014

Crane, 2011

Subramoney, 2010

Pooled data

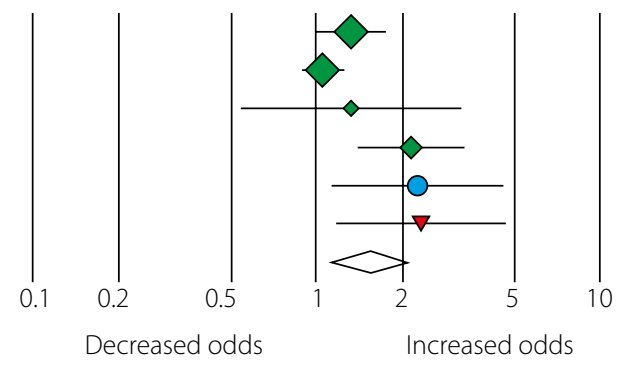

HIC LOW QA
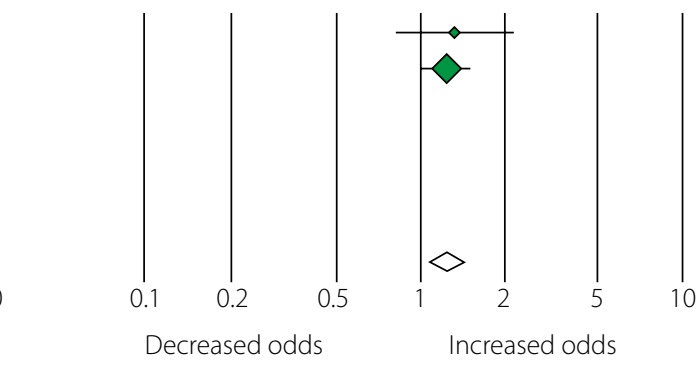

Decreased odds

Increased odds

$\nabla$ LMIC High QA

COR - crude odds ratio, aOR - adjusted odds ratio, *adjusted odds ratios included in high-quality analysis, $\mathrm{Cl}$ - confidence interval, QA - quality assessment, HIC - high-income country, LMIC - low- or middle-income country

FIG. 4. Stillbirth forest plot

tend to be associated with increased daily SHS exposure [56]. The relative risks reported for adverse reproductive outcomes among mothers who smoke during pregnancy are not dramatically different from the relative risks found here for SHS exposure. Studies of maternal smok- ing associated with low-birth weight and orofacial clefts have reported odds ratios up to 2.5 , which is higher than the effects found in this analysis, but results are similar for PTB and stillbirth. Thus, it is reasonable to expect that the far greater prevalence of SHS exposure during pregnan- 


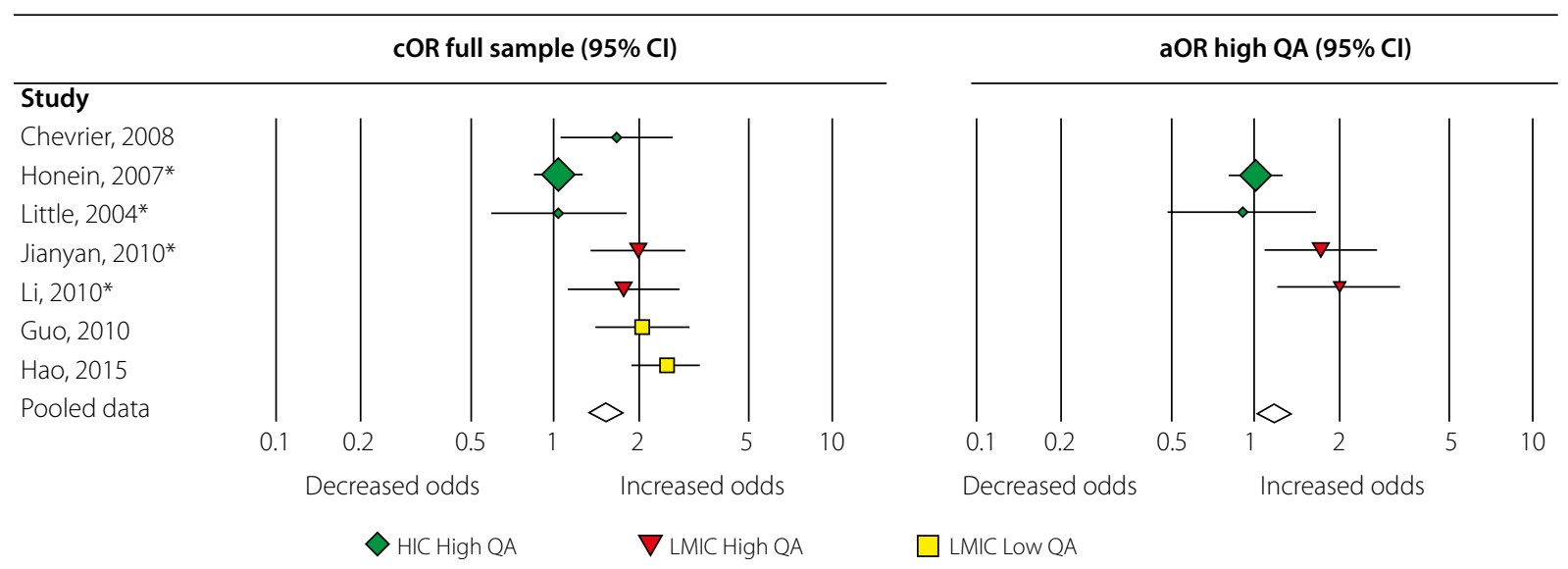

COR - crude odds ratio, aOR - adjusted odds ratio, *adjusted odds ratios included in high-quality analysis, CI - confidence interval, QA - quality assessment, HIC - high-income country, LMIC - low- or middle-income country

FIG. 5. Cleft lip with or without cleft palate forest plot

cy may have a greater impact, particularly in those countries where smoking is very low among women but high among men [57].

In 2013, the World Health Organisation published recommendations for the prevention and management of tobacco use and SHS exposure in pregnancy, noting that pregnancy is often a window of opportunity to intervene with women and their families [58]. However, evidence on effective interventions is limited, especially in LMICs. A systematic review found that clinical interventions delivered in prenatal care settings appear to reduce SHS exposure, but that more rigorous studies, using biochemical validation, are needed [59]. A multi-method study from Indonesia found high levels of exposure to SHS as well as high levels of awareness about risks to children, but very low prevalence of household indoor smoking rules. While women expressed a low sense of self-efficacy about persuading their husbands to quit smoking in their homes, community-wide smoking bans had substantial support [60]. Evidence from HICs has shown that smoke-free policies have led to reductions in adverse maternal and child health outcomes [61, 62]. Additionally, a recent study in 23 European Union countries found that increases in the median price of cigarettes were associated with reductions in infant mortality between 2004 and 2014, probably because of reductions in smoking and secondhand smoke exposure [63]. However, more research is needed to develop and evaluate interventions to address SHS exposure during pregnancy in LMICs in the context of cultural and socioeconomic barriers [4].

Limitations of the current study include the high heterogeneity $\left(I^{2}>75 \%\right)$ of some of the analyses, which may suggest a lack of generalisability. Because high heterogeneity was more common in the MIC analyses than the HIC analyses, it may reflect differences in smoking prevalence and smoking intensity across MICs; it could also reflect differences in exposure assessment. Notably, heterogeneity was high in all studies on cleft palate in MICs although all five studies were conducted in China. It is possible that the relatively low quality of these five studies impacted the risk estimates for cleft palate.

Additional limitations relate to the measurement of reproductive outcomes and SHS exposure in the studies used for this analysis. For outcomes of preterm birth, almost all studies from MICs only used the last menstrual date to determine whether the birth was preterm, and most studies from MICs did not confirm gestational age when looking at LBW. Preterm birth and stillbirth can be difficult to confirm in low-resource settings because of the difficulty of accurately assessing gestational age. Collecting data about birth outcomes via birth records also constitutes another limitation of this meta-analysis. Birth outcomes may not be reported if the birth does not occur in a clinic, and high-risk pregnancies are more often referred to clinics for births in some settings, so the rates may be overestimated for certain outcomes. Lastly, in most studies SHS exposure during pregnancy was estimated by self-reported household smoking status without biochemical verification. It is especially challenging to precisely assess SHS exposure from self-report because exposure may be influenced not only by frequency of smoking but also by conditions in the home and the amount of time spent in the home.

This study demonstrates that SHS exposure poses a serious threat for maternal and child health in LMICs as well as HICs. In fact, the magnitude of the risk for birth outcomes included in the analysis was greater among the MIC studies than the HIC studies. More high-quality studies of SHS exposure during pregnancy are needed from LMICs to better characterise the magnitude of the risk and understand the factors behind these 
differences. Additionally, more comprehensive monitoring of SHS exposure during pregnancy and evaluation of potential interventions are needed to effectively address the adverse impact of SHS exposure during pregnancy in parts of the world where the burden is greatest.

\section{ACKNOWLEDGMENTS}

This project has been funded in part with federal funds from the National Cancer Institute, National Institutes of Health, U.S. Department of Health and Human Services; this analysis was funded partially under Contract No. HHSN261201000043C and Contract No. HHSN261201700004I. We thank Lucinda England, MD, MSPH for providing scientific review, and Juliette Kendrick, MD, Jennita Reefhuis, $\mathrm{PhD}$, Marty Kharrazi, $\mathrm{PhD}$, and Peggy Honein, PhD, MPH for their subject expertise on adapting the quality assessment tool.

\section{DISCLOSURE}

The authors report no conflict of interest.

\section{References}

1. U.S. Department of Health and Human Services. The health consequences of smoking-50 years of progress: a report of the Surgeon General. Atlanta: U.S. Department of Health and Human Services, Centers for Disease Control and Prevention, National Center for Chronic Disease Prevention and Health Promotion, Office on Smoking and Health; 2014. Available from: https://www.ncbi.nlm.nih.gov/books/NBK179276/pdf/ Bookshelf_NBK179276.pdf (accessed: 11 March 2019).

2. World Health Organization. WHO report on the global tobacco epidemic, 2017: monitoring tobacco use and prevention policies. Geneva: World Health Organization; 2017. Available from: https://www.who.int/tobacco/global_report/2017/en/ (accessed: 11 March 2019).

3. Asma S, Mackay J, Song SY, et al. The GATS atlas. Atlanta: CDC Foundation; 2015. Available from: http://gatsatlas.org/ downloads/GATS-whole-book-12.pdf (accessed: 11 March 2019).

4. Nichter M, Greaves L, Bloch M, et al. Tobacco use and secondhand smoke exposure during pregnancy in low- and middle-income countries: the need for social and cultural research. Acta Obstet Gynecol Scand 2010; 89: 465-477.

5. World Health Organization. Tobacco Free Initiative (TFI) Global Adult Tobacco Survey [Website] [multiple years]. Available from: http://www.who.int/tobacco/surveillance/survey/ gats/en (accessed: 11 March 2019).

6. Caleyachetty R, Tait CA, Kengne AP, et al. Tobacco use in pregnant women: analysis of data from Demographic and Health Surveys from 54 low-income and middle-income countries. Lancet Glob Health 2014; 2: e513-520.

7. Liu T, Chen W. Meta analysis of effects on maternal passive smoking during pregnancy on fetal low birth weight. Wei Sheng Yan Jiu 2009; 38: 5677-5681.

8. Windham GC, Eaton A, Hopkins B. Evidence for an association between environmental tobacco smoke exposure and birth- weight: a meta-analysis and new data. Paediatr Perinat Epidemiol 1999; 13: 35-57.

9. Leonardi-Bee J, Smyth A, Britton J, Coleman T. Environmental tobacco smoke and fetal health: systematic review and meta-analysis. Arch Dis Child Fetal Neonatal Ed 2008; 93: F351-361.

10. Salmasi G, Grady R, Jones J, McDonald SD; Knowledge Synthesis Group. Environmental tobacco smoke exposure and perinatal outcomes: a systematic review and meta-analyses. Acta Obstet Gynecol Scand 2010; 89: 423-441.

11. Sabbagh HJ, Hassan MH, Innes NP, et al. Passive smoking in the etiology of non-syndromic orofacial clefts: a systematic review and meta-analysis. PLoS One 2015; 10: e0116963.

12. Leonardi-Bee J, Britton J, Venn A. Secondhand smoke and adverse fetal outcomes in nonsmoking pregnant women: a meta-analysis. Pediatrics 2011; 127: 734-741.

13. Nieuwenhuijsen MJ, Dadvand P, Grellier J, et al. Environmental risk factors of pregnancy outcomes: a summary of recent meta-analyses of epidemiological studies. Environ Health 2013; 12: 6.

14. Abu-Baker NN, Haddad L, Savage C. The influence of secondhand smoke exposure on birth outcomes in Jordan. Int J Environ Res Public Health 2010; 7: 616-634.

15. Abusalah A, Gavana M, Haidich AB, et al. Low birth weight and prenatal exposure to indoor pollution from tobacco smoke and wood fuel smoke: a matched case-control study in Gaza Strip. Matern Child Health J 2012; 16: 1718-1727.

16. Andriani $\mathrm{H}, \mathrm{Kuo} \mathrm{H}$. Adverse effects of parental smoking during pregnancy in urban and rural areas. BMC Pregnancy Childbirth 2014; 14: 414-428.

17. Crane JM, Keough M, Murphy P, et al. Effects of environmental tobacco smoke on perinatal outcomes: a retrospective cohort study. BJOG 2011; 118: 865-871.

18. Ghosh JK, Wilhelm M, Ritz B. Effects of residential indoor air quality and household ventilation on preterm birth and term low birth weight in Los Angeles County, California. Am J Public Health 2013; 103: 686-694.

19. Khader YS, Al-Akour N, Alzubi IM, Lataifeh I. The association between second hand smoke and low birth weight and preterm delivery. Matern Child Health J 2011; 15: 453-459.

20. Khattar D, Awasthi S, Das V. Residential environmental tobacco smoke exposure during pregnancy and low birth weight of neonates: case control study in a public hospital in Lucknow, India. Indian Pediatr 2013; 50: 134-138.

21. Ko TJ, Tsai LY, Chu LC, et al. Parental smoking during pregnancy and its association with low birth weight, small for gestational age, and preterm birth offspring: a birth cohort study. Pediatr Neonatol 2014; 55: 20-27.

22. Metgud CS, Naik VA, Mallapur MD. Factors affecting birth weight of a newborn - a community based study in rural Karnataka, India. PLoS One 2012; 7: e40040.

23. Norsaadah B, Salinah O. The effect of second-hand smoke exposure during pregnancy on the newborn weight in Malaysia. Malays J Med Sci 2014; 21: 44-53.

24. Subramoney S, d'Espaignet ET, Gupta PC. Higher risk of stillbirth among lower and middle income women who do not use 
tobacco, but live with smokers. Acta Obstet Gynecol Scand 2010; 89: 572-577.

25. Wahabi HA, Alzeidan RA, Fayed AA, et al. Effects of secondhand smoke on the birth weight of term infants and the demographic profile of Saudi exposed women. BMC Public Health 2013; 13: 341-346.

26. Ashford KB, Hahn E, Hall L, et al. The effects of prenatal secondhand smoke exposure on preterm birth and neonatal outcomes. J Obstet Gynecol Neonatal Nurs 2010; 39: 525-535.

27. Ion RC, Wills AK, Bernal AL. Environmental tobacco smoke exposure in pregnancy is associated with earlier delivery and reduced birth weight. Reprod Sci 2015; 22: 1603-1611.

28. Luo YJ, Wen XZ, Ding P, et al. Interaction between maternal passive smoking during pregnancy and CYP1A1 and GSTs polymorphisms on spontaneous preterm delivery. PLoS One 2012; 7: e49155.

29. Mojibyan M, Karimi M, Bidaki R, et al. Exposure to second-hand smoke during pregnancy and preterm delivery. Int J High Risk Behav Addict 2013; 1: 149-153.

30. Qiu J, He X, Cui H, et al. Passive smoking and preterm birth in urban China. Am J Epidemiol 2014; 180: 94-102.

31. Gardosi J, Madurasinghe V, Williams M, et al. Maternal and fetal risk factors for stillbirth: population based study. BMJ 2013; 346: f108.

32. Hyland A, Piazza KM, Hovey KM, et al. Associations of lifetime active and passive smoking with spontaneous abortion, stillbirth and tubal ectopic pregnancy: a cross-sectional analysis of historical data from the Women's Health Initiative. Tob Control 2015; 24: 328-335.

33. Varner MW, Silver RM, Rowland Hogue CJ, et al. Association between stillbirth and illicit drug use and smoking during pregnancy. Obstet Gynecol 2014; 123: 113-125.

34. Chevrier C, Bahuau M, Perret C, et al. Genetic susceptibilities in the association between maternal exposure to tobacco smoke and the risk of nonsyndromic oral cleft. Am J Med Genet A 2008; 146A: 2396-2406.

35. Hao Y, Tian S, Jiao X, et al. Association of parental environmental exposures and supplementation intake with risk of nonsyndromic orofacial clefts: a case-control study in Heilongjiang Province, China. Nutrients 2015; 7: 7172-7184.

36. Fantuzzi G, Aggazzotti G, Righi E, et al. Preterm delivery and exposure to active and passive smoking during pregnancy: a case-control study from Italy. Paediatr Perinat Epidemiol 2007; 21: 194-200.

37. Goel P, Radotra A, Singh I, et al. Effects of passive smoking on outcome in pregnancy. J Postgrad Med 2004; 50: 12-16.

38. Guo Z, Huang C, Ding K, et al. Transforming growth factor beta- 3 and environmental factors and cleft lip with/without cleft palate. DNA Cell Biol 2010; 29: 375-380.

39. Honein MA, Rasmussen SA, Reefhuis J, et al. Maternal smoking and environmental tobacco smoke exposure and the risk of orofacial clefts. Epidemiology 2007; 18: 226-233.

40. Jaddoe VW, Troe EJ, Hofman A, et al. Active and passive maternal smoking during pregnancy and the risks of low birthweight and preterm birth: the Generation R Study. Paediatr Perinat Epidemiol 2008; 22: 162-171.
41. Jia ZL, Shi B, Chen CH, et al. Maternal malnutrition, environmental exposure during pregnancy and the risk of non-syndromic orofacial clefts. Oral Dis 2011; 17: 584-589.

42. Jianyan L, Zeqiang G, Yongjuan C, et al. Analysis of interactions between genetic variants of BMP4 and environmental factors with nonsyndromic cleft lip with or without cleft palate susceptibility. Int J Oral Maxillofac Surg 2010; 39: 50-56.

43. Kharrazi M, DeLorenze GN, Kaufman FL, et al. Environmental tobacco smoke and pregnancy outcome. Epidemiology 2004; 15: 660-670.

44. Krstev S, Marinkovic J, Simic S, et al. The influence of maternal smoking and exposure to residential ETS on pregnancy outcomes: a retrospective national study. Matern Child Health J 2013; 17: 1591-1598.

45. Li Z, Liu J, Ye R, et al. Maternal passive smoking and risk of cleft lip with or without cleft palate. Epidemiology 2010; 21: 240-242.

46. Little J, Cardy A, Arslan MT, et al.; United Kingdom-based case-control study. Smoking and orofacial clefts: A United Kingdom-based case-control study. Cleft Palate Craniofacial J 2004; 41: 381-386.

47. Ojima $\mathrm{T}$, Uehara R, Watanabe $\mathrm{M}$, et al. Population attributable fraction of smoking to low birth weight in Japan. Pediatr Int 2004; 46: 264-267.

48. Pogodina C, Huber LR, Racine EF, Platonova E. Smoke-free homes for smoke-free babies: the role of residential environmental tobacco smoke on low birth weight. J Community Health 2009; 34: 376-382.

49. Uncu Y, Ozcakir A, Ercan I, et al. Pregnant women quit smoking; what about fathers? Survey study in Bursa Region, Turkey. Croat Med J 2005; 46: 832-837.

50. Ward C, Lewis S, Coleman T. Prevalence of maternal smoking and environmental tobacco smoke exposure during pregnancy and impact on birth weight: retrospective study using Millennium Cohort. BMC Public Health 2007; 7: 81-86.

51. Wdowiak A, Wiktor H, Wdowiak L. Maternal passive smoking during pregnancy and neonatal health. Ann Agric Environ Med 2009; 16: 309-312.

52. Wu T, Hu Y, Chen C, et al. Passive smoking, metabolic gene polymorphisms, and infant birth weight in a prospective cohort study of Chinese women. Am J Epidemiol 2007; 166: 313-322.

53. Bloch M, Althabe F, Onyamboko M, et al. Tobacco use and secondhand smoke exposure during pregnancy: an investigative survey of women in 9 developing nations. Am J Public Health 2008; 98: 1833-1840.

54. Reece S, Morgan C, Parascandola M, Siddiqi K. Secondhand smoke exposure during pregnancy: a cross-sectional analysis of data from Demographic and Health Survey from 30 lowincome and middle-income countries. Tob Control 2019; 28: $420-426$.

55. Öberg M, Jaakkola MS, Woodward A, et al. Worldwide burden of disease from exposure to second-hand smoke: a retrospective analysis of data from 192 countries. Lancet 2011; 377: 139-146.

56. Fischer F, Minnwegen M, Kaneider U, Kraemer A, Khan MM. Prevalence and determinants of secondhand smoke exposure among women in Bangladesh, 2011. Nicotine Tob Res 2015; 17: 58-65. 
57. U.S. Department of Health and Human Services. The health consequences of smoking: a report of the Surgeon General. Atlanta: U.S. Department of Health and Human Services, Centers for Disease Control and Prevention, National Center for Chronic Disease Prevention and Health Promotion, Office on Smoking and Health; 2004. Available from: https://www.cdc. gov/tobacco/data_statistics/sgr/2004/complete_report/index. htm (accessed: 13 March 2019).

58. World Health Organization. WHO recommendations for the prevention and management of tobacco use and second-hand smoke exposure in pregnancy. World Health Organization, Geneva 2013. Available from: http://apps.who.int/iris/bitstream/handle/10665/94555/9789241506076_eng.pdf?sequence $=1$ (accessed: 12 March 2019).

59. Tong VT, Dietz PM, Rolle IV, et al. Clinical interventions to reduce secondhand smoke exposure among pregnant women: a systematic review. Tob Control 2015; 24: 217-233.

60. Nichter M, Nichter M, Siwi Padmawati R, Ng N. Developing a smoke free household initiative: an Indonesian case study. Acta Obstet Gynecol Scand 2010; 89: 578-581.

61. Been JV, Nurmatov UB, Cox B, et al. Effect of smoke-free legislation on perinatal and child health: a systematic review and meta-analysis. Lancet 2014; 383: 1549-1560.

62. Been JV, Mackay DF, Millett C, et al. Impact of smoke-free legislation on perinatal and infant mortality: a national quasiexperimental study. Sci Rep 2015; 5: 13020.

63. Filippidis FT, Laverty AA, Hone T, et al. Association of cigarette price differentials with infant mortality in 23 European Union countries. JAMA Pediatr 2017; 171: 1100-1106.

\section{AUTHOR'S CONTRIBUTIONS}

MP, ALK, and JB prepared the research concept and design, collected data, wrote the article, revised the article and finally approved it. 